\title{
A new finite element method for strain gradient theories and applications to fracture analyses
}

\author{
Yueguang Wei* \\ LNM, Institute of Mechanics, Chinese Academy of Sciences, Beijing 100080, China \\ Received 23 June 2005; accepted 2 March 2006 \\ Available online 17 April 2006
}

\begin{abstract}
A new compatible finite element method for strain gradient theories is presented. In the new finite element method, pure displacement derivatives are taken as the fundamental variables. The new numerical method is successfully used to analyze the simple strain gradient problems - the fundamental fracture problems. Through comparing the numerical solutions with the existed exact solutions, the effectiveness of the new finite element method is tested and confirmed. Additionally, an application of the Zienkiewicz-Taylor C1 finite element method to the strain gradient problem is discussed. By using the new finite element method, plane-strain mode I and mode II crack tip fields are calculated based on a constitutive law which is a simple generalization of the conventional $J_{2}$ deformation plasticity theory to include strain gradient effects. Three new constitutive parameters enter to characterize the scale over which strain gradient effects become important. During the analysis the general compressible version of Fleck-Hutchinson strain gradient plasticity is adopted. Crack tip solutions, the traction distributions along the plane ahead of the crack tip are calculated. The solutions display the considerable elevation of traction within the zone near the crack tip.
\end{abstract}

(c) 2006 Elsevier Masson SAS. All rights reserved.

Keywords: Finite element method; Strain gradient theory; Crack tip fields

\section{Introduction}

Recently, with advancements in experimental technique and measuring precision, many experimental evidences have displayed that at the micron or sub-micron scales the metal material behaves with a significantly higher strength than that when it is at the macro-scale. Such a difference of the mechanical behaviors between the micron scale and the macro-scale is often referred to as the size effect. For example, in the micro-indentation tests for metals (Stelmashenko et al., 1993; Ma and Clarke, 1995; McElhaney et al., 1998; Wei et al., 2001), the measured hardness values increase as the indent depth decreases from microns to sub-micron. Size effect phenomena have also observed in the micro-torsion test for copper wire (Fleck et al., 1994), and in the thin-beam bending test for metals (Stolken and Evans, 1998), as well as in the interfacial adhesion experiments for the metal/ceramic systems (Bagchi and Evans, 1996; Lipkin et al., 1998), etc. About fracture of solids, attempts to link macroscopic fracture behavior to atomistic fracture processes in ductile metals are frustrated by the inability of conventional elastic-plastic theories to adequately model stress-strain

\footnotetext{
* Tel.: +861062648721 ; fax: +861062561284 .

E-mail address: ywei@1nm.imech.ac.cn (Y. Wei).
} 
behavior at the small scales required in crack tip models. Adding to the complications of the atomistic separation processes and interactions of individual dislocations with the crack tip, is the complication of an intermediate region lying between the tip and the outer plastic zone within which stresses are almost certainly much higher than implied by conventional elastic-plastic theory. An obvious reason why the conventional elastic-plastic theory cannot simulate the size effect successfully is that the conventional theory does not include any length parameters, with which the difference of behaviors of solids in macro- and micro-scales can be distinguished.

In order to describe and model the size effects, several strain gradient theories have been presented (Fleck and Hutchinson, 1993; 1997; Aifantis, 1984; Gao et al., 1999; Chen and Wang, 2001; etc.). In the strain gradient theories, several length parameters are included, and through them, the size effects are predicted and characterized. However, since the strain gradient terms are included in the constitutive equations and the displacement gradient terms appear in the boundary conditions, the considerable complications and difficulties are come out in solving the related problems (see Engel et al., 2002; Shu et al., 1999; Matsushima et al., 2002). Generally, the conventional displacement-based finite element method fails to simulate the strain gradient effect. Although some simulation and modeling methods have been presented in last several years for studying the micro-indentation tests (Shu and Fleck, 1998; Begley and Hutchinson, 1998; Wei et al., 2001), the stationary and growing crack tip fields (Xia and Hutchinson, 1996; Wei and Hutchinson, 1997; Zhang et al., 1998; Huang et al., 1999; Shi et al., 2000), as well as the plate (or beam) bending problem (Engel et al., 2002), it is still important and a tough challenge to develop an effective finite element methods for the strain gradient theories (Engel et al., 2002). In the present paper, a new finite element method for strain gradient theories will be presented. In developing the special finite element methods, it is important to test the effectiveness of the methods through applying them to the analyses of some typical problems which have had the closed-form solutions. It is worth noting that, Huang and his collaborators have solved the several fundamental elastic fracture problems based on the strain gradient theories of Fleck and Hutchinson $(1993 ; 1997)$, and obtained a series of the closed-form analytical solutions (Zhang et al., 1998; Shi et al., 2000). These basic solutions will be used to check the effectiveness of the new finite element methods in the present investigations.

In the new finite element method, the displacement derivatives are taken as the fundamental variables. Through applying the new finite element method to the analyses for the fundamental fracture problems (elastic strain gradient problems: Mode I, Mode II and Mode III), the effectiveness of the new finite element method is tested and confirmed. Moreover, the effectiveness of the Zienkiewicz-Taylor $\mathrm{C} 1$ finite element method in application to the strain gradient problems is also discussed. Furthermore, the new finite element method is used to analyze the plane strain and strain gradient elastic-plastic fracture problems. The crack tip fields, the traction distributions along the plane ahead of the crack tip, are studied and the size effect in microscopic fracture is analyzed. In the present research, the adopted strain gradient plasticity theory is the generalized compressible deformational theory of Fleck-Hutchinson version (Fleck and Hutchinson, 1997) derived by Wei (2001).

\section{Problem formulations}

\subsection{Fleck-Hutchinson strain gradient plasticity theory (the generalized compressible deformational theory)}

The general compressible deformational theory of Fleck-Hutchinson strain gradient plasticity (Fleck and Hutchinson, 1997) has been derived by Wei (2001). A brief description is outlined as follows.

The definitions of the strain and strain gradient are defined by

$$
\varepsilon_{i j}=\frac{1}{2}\left(u_{i, j}+u_{j, i}\right)=\varepsilon_{i j}^{\mathrm{e}}+\varepsilon_{i j}^{\mathrm{p}}, \quad \eta_{i j k}=u_{k, i j}=\eta_{i j k}^{\mathrm{e}}+\eta_{i j k}^{\mathrm{p}},
$$

The elastic strains $\varepsilon_{i j}^{\mathrm{e}}$ and elastic strain gradients $\eta_{i j k}^{\mathrm{e}}$ are related to the stress $\sigma_{i j}$ and the higher-order stress $\tau_{i j k}$ through defining an elastic strain energy density,

$$
W^{\mathrm{e}}=E\left(\frac{v}{2(1+v)(1-2 v)} \varepsilon_{k k}^{\mathrm{e} 2}+\frac{1}{2(1+v)} \varepsilon_{i j}^{\mathrm{e}} \varepsilon_{i j}^{\mathrm{e}}+\sum_{I=1}^{4} L_{I}^{\mathrm{e} 2} \eta_{i j k}^{\mathrm{e}(I)} \eta_{i j k}^{\mathrm{e}(I)}\right)
$$

and by using the conjugate relations

$$
\sigma_{i j}=\partial W^{\mathrm{e}} / \partial \varepsilon_{i j}^{\mathrm{e}}, \quad \tau_{i j k}=\partial W^{\mathrm{e}} / \partial \eta_{i j k}^{\mathrm{e}},
$$


where, $E$ and $v$ are the Young's modulus and Poisson ratio, respectively, $L_{I}^{\mathrm{e}}(I=1,4)$ are the elastic length parameters, $\eta_{i j k}^{\mathrm{e}(I)}(I=1,3)$ are the deviatoric part of $\eta_{i j k}^{\mathrm{e}}, \eta_{i j k}^{\mathrm{e}(4)}$ is the hydrostatic part of $\eta_{i j k}^{\mathrm{e}}$ concerning the volume deformation. Assuming that the contribution to the strain energy density from the mixed part of both the hydrostatic and deviatoric parts of the strain gradient invariants, $\eta_{i j k}^{\mathrm{e}(4)} \eta_{i j k}^{\mathrm{e}(3)}$, can be neglected, as in author's another paper (Wei and Hutchinson, 1997), so we immediately arrive at the elastic strain energy density expression, Eq. (2).

For $J_{2}$ deformation theory, the effective strain and effective stress considering the strain gradient effects are defined by

$$
\Xi=\sqrt{\frac{2}{3} \varepsilon_{i j}^{\prime} \varepsilon_{i j}^{\prime}+\sum_{I=1}^{3} L_{I}^{2} \eta_{i j k}^{(I)} \eta_{i j k}^{(I)}}, \quad \Sigma=\sqrt{3 J_{2}}=\sqrt{\frac{3}{2} \sigma_{i j}^{\prime} \sigma_{i j}^{\prime}+\sum_{I=1}^{3} L_{I}^{-2} \tau_{i j k}^{(I)} \tau_{i j k}^{(I)}}
$$

with $L_{I}(I=1,3)$ as the plastic length parameters, where

$$
\eta_{i j k}^{(I)}=T_{i j k l m n}^{(I)} \eta_{l m n}, \quad \tau_{i j k}^{(I)}=T_{i j k l m n}^{(I)} \tau_{l m n},
$$

$T_{i j k l m n}^{(I)}(I=1,4)$ is the projection tensor of the strain gradients, and the detailed expressions have been presented by Wei and Hutchinson (1997). Thus, the plastic strains and strain gradients can be expressed

$$
\varepsilon_{i j}^{\mathrm{p}}=\frac{3}{2 h^{\mathrm{p}}} \frac{\partial J_{2}}{\partial \sigma_{i j}}=\frac{3}{2 h^{\mathrm{p}}} \sigma_{i j}^{\prime}, \quad \eta_{i j k}^{\mathrm{p}}=\frac{3}{2 h^{\mathrm{p}}} \frac{\partial J_{2}}{\partial \tau_{i j k}}=\frac{1}{h^{\mathrm{p}}} \sum_{I=1}^{3} L_{I}^{-2} T_{i j k l m n}^{(I)} \tau_{l m n},
$$

where

$$
h^{\mathrm{p}}=\Sigma /(\Xi-\Sigma / E),
$$

$h^{\mathrm{p}}$ is the plastic modulus. Considering the power-law hardening material,

$$
\Xi=\Sigma / E, \quad \Sigma \leqslant \sigma_{Y} ; \quad \Xi=\left(\sigma_{Y} / E\right)\left(\Sigma / \sigma_{Y}\right)^{1 / N}, \quad \Sigma>\sigma_{Y}
$$

one has

$$
h^{\mathrm{p}}=E\left\{\left(\Sigma / \sigma_{Y}\right)^{1 / N-1}-1\right\}^{-1},
$$

where $\sigma_{Y}$ is material yield strength, and $N$ is the strain hardening exponent.

By using the relations from (1) to (9) and the normality of projection tensors, the general compressible form of the deformational theory of strain gradient plasticity can be derived out

$$
\begin{aligned}
\sigma_{i j} & =\frac{E}{1+v+\frac{3}{2} E / h^{\mathrm{p}}} \varepsilon_{i j}+\frac{1}{3}\left(\frac{E}{1-2 v}-\frac{E}{1+v+\frac{3}{2} E / h^{\mathrm{p}}}\right) \varepsilon_{k k} \delta_{i j}, \\
\tau_{i j k} & =2 E\left\{\sum_{I=1}^{3} \frac{L_{I}^{2}}{L_{I}^{2} / L_{I}^{\mathrm{e} 2}+2 E / h^{\mathrm{p}}} T_{i j k l m n}^{(I)}+L_{4}^{\mathrm{e} 2} T_{i j k l m n}^{(4)}\right\} \eta_{l m n} .
\end{aligned}
$$

For strain gradient elasticity, the constitutive relations can be simplified through letting $E / h^{\mathrm{p}}=0$ in Eq. (10), or directly from (2) and (3), one can derive

$$
\sigma_{i j}=\frac{E}{1+v} \varepsilon_{i j}+\frac{E v}{(1-2 v)(1+v)} \varepsilon_{k k} \delta_{i j}, \quad \tau_{i j k}=2 E\left\{\sum_{I=1}^{4} L_{I}^{\mathrm{e}^{2}} T_{i j k l m n}^{(I)}\right\} \eta_{l m n} .
$$

In formulas (10) and (11), $L_{I}^{\mathrm{e}}(I=1,4)$ and $L_{I}(I=1,3)$ are the length-scale parameters of strain gradient elasticity and plasticity, respectively. From (4) (or (2)), $L_{I}$ (or $\left.L_{I}^{\mathrm{e}}\right)(I=1,3)$ characterize the strength of energy density contributed from the deviation part of higher-order strains and stresses, and $L_{4}^{\mathrm{e}}$ characterizes strength of the elastic energy density contributed from hydrostatic part of higher-order strains and stresses. From the discussion by Fleck and Hutchinson (1997), for more general solid which is dependent on both stretch and rotation gradients (SG theory), there is an approximate relation among the parameters:

$$
L_{1}=L, \quad L_{2}=\frac{1}{2} L, \quad L_{3}=\sqrt{\frac{5}{24}} L .
$$


For couple stress theory (Fleck and Hutchinson, 1993), the relation among the parameters is

$$
L_{1}=0, \quad L_{2}=\frac{1}{2} L, \quad L_{3}=\sqrt{\frac{5}{24}} L .
$$

Similarly, the discussion and corresponding results, (12) and (13), are also suitable for strain gradient elasticity case. Previous researches by author have shown that the solution is insensitive to the value of ratio $L^{\mathrm{e}} / L$ within the region $0<L^{\mathrm{e}} / L<1$ (Wei and Hutchinson, 1997). Thus, in the present study let $L^{\mathrm{e}} / L=0.5$, in addition, take $L_{4}^{\mathrm{e}}=L^{\mathrm{e}} / 2$.

Clearly, the constitutive relations (10) will degenerate to the conventional elastic-plastic constitutive relation for $L \rightarrow 0$.

\subsection{Variational relations}

Frequently, equilibrium equations are replaced by variational relation in using the finite element method. Different forms of the variational relations correspond to the different finite element methods. In the variational relation for conventional elastic-plastic theory, the displacement components are taken as the fundamental variables with the displacements as the nodal variables in the conventional finite element method. For the strain gradient theory as discussed above, however, a proper form of the variational relation needs to be discussed, and an effective finite element method needs to be studied. A candidate variational relation form was given by Fleck and Hutchinson (1997), which is dependent on the two types of variables, the displacements and the displacement derivatives. Naturally, the corresponding finite element method which is similar to the Zienkiewicz-Taylor C1 continuity element scheme by taking both displacements and displacement derivatives as the nodal variables is considered first. Moreover, in the present study, a new form of variational relation will be considered, and a new finite element method will be presented based on the new form of variational relation. The effectiveness of both finite element methods will be tested through applications of them to some fundamental strain gradient problems which have the closed-form exact solutions and through comparing the numerical solutions with the existed exact solutions. In the following analyses, two kinds of variational relations will be considered.

\subsubsection{Taking pure displacement derivatives as the fundamental variables}

For the purpose of developing a new finite element method, we consider a new form of variational equation by one-step integration

$$
\begin{aligned}
\int_{V}\left(\sigma_{i j} \delta \varepsilon_{i j}+\tau_{i j k} \delta \eta_{i j k}\right) \mathrm{d} V & =\int_{V}\left\{\sigma_{i j} \delta \frac{\partial u_{i}}{\partial x_{j}}+\tau_{i j k} \delta \frac{\partial^{2} u_{k}}{\partial x_{i} \partial x_{j}}\right\} \mathrm{d} V \\
& =\int_{V}\left\{\sigma_{l j}-\tau_{i j l, i}\right\} \delta \frac{\partial u_{l}}{\partial x_{j}} d V+\int_{S} n_{i} \tau_{i j l} \delta \frac{\partial u_{l}}{\partial x_{j}} \mathrm{~d} S .
\end{aligned}
$$

Eq. (14) can be further expressed as

$$
\int_{V}\left\{\sigma_{i j} \delta U_{i j}+\tau_{i j k} \delta \frac{\partial U_{k j}}{\partial x_{i}}\right\} \mathrm{d} V=\int_{V} f_{i j}^{B} \delta U_{i j} \mathrm{~d} V+\int_{S} M_{j i} \delta U_{i j} \mathrm{~d} S,
$$

where

$$
U_{i j}=\frac{\partial u_{i}}{\partial x_{j}}, \quad f_{i j}^{\mathrm{B}}=\sigma_{i j}-\tau_{k j i, k}, \quad M_{j i}=n_{k} \tau_{k j i} .
$$

$f_{i j}^{\mathrm{B}}$ and $M_{i j}$ are taken as the generalized body force and the generalized surface force, respectively. $U_{i j}$ is the generalized displacement. The physical significance of the new variational equation (15) can be understood as that the variation of total strain energy is equal to the variation of the work done by the generalized body force and the generalized surface force with respect to the generalized displacement. With the new denotation in (16), the constitutive relations, (10) can be briefly rewritten as

$$
\sigma_{i j}=D_{i j k l} U_{k l}, \quad \tau_{i j k}=C_{i j k l m n} U_{n l, m} .
$$

In (15) and (16), the fundamental variables are the pure displacement derivatives $U_{i j}$. 


\subsubsection{Taking both displacements and displacement derivatives as the fundamental variables}

The variational relation based on both the displacements and displacement derivatives has been derived by Fleck and Hutchinson (1997). For comparison and for conveniently discussing the Zienkiewicz-Taylor C1 finite element method in the following sections, the result is given here

$$
\int_{V}\left(\sigma_{i j} \delta \varepsilon_{i j}+\tau_{i j k} \delta \eta_{i j k}\right) \mathrm{d} V=\int_{V} f_{k} \delta u_{k} \mathrm{~d} V+\int_{S} t_{k} \delta u_{k} \mathrm{~d} S+\int_{S} r_{k}\left(\widehat{D} \delta u_{k}\right) \mathrm{d} S .
$$

Based on Eq. (18), the traction and toque on $S$ surface have been derived by Fleck and Hutchinson as

$$
t_{k}=n_{i}\left(\sigma_{i k}-\frac{\partial \tau_{i j k}}{\partial x_{j}}\right)+n_{i} n_{j} \tau_{i j k}\left(D_{p} n_{p}\right)-D_{j}\left(n_{i} \tau_{i j k}\right), \quad r_{k}=n_{i} n_{j} \tau_{i j k} .
$$

The operators $\widehat{D}$ and $D_{j}$ in (18) and (19) are defined as

$$
D_{j}=\partial / \partial x_{j}-n_{j} n_{k} \partial / \partial x_{k}, \quad \widehat{D}=n_{k} \partial / x_{k},
$$

where $n_{i}$ in Eqs. (18)-(20) is the direction cosine of $S$ surface. In the variational relation (18) both the displacements and the displacement derivatives are taken as the fundamental variables.

\section{A new finite element method}

The variational relations (see (15) and (16)) imply that the pure displacement derivatives can be taken as the fundamental variables in finite element method. Following the point, a new finite element method for the strain gradient theory can be presented.

For convenience, 3-noded triangular element is used in the present study. As usual, the following area coordinates $\left(f_{i}\right)$ and their derivatives are adopted

$$
\begin{aligned}
& \left(f_{1}, f_{2}, f_{3}\right)=\left(\Delta_{1} / \Delta, \Delta_{2} / \Delta, \Delta_{3} / \Delta\right), \quad\left(f_{1 x}, f_{2 x}, f_{3 x}\right)=\left(b_{1} / \Delta, b_{2} / \Delta, b_{3} / \Delta\right), \\
& \left(f_{1 y}, f_{2 y}, f_{3 y}\right)=\left(c_{1} / \Delta, c_{2} / \Delta, c_{3} / \Delta\right),
\end{aligned}
$$

where

$$
b_{1}=y_{2}-y_{3}, \quad b_{2}=y_{3}-y_{1}, \quad b_{3}=y_{1}-y_{2}, \quad c_{1}=x_{3}-x_{2}, \quad c_{2}=x_{1}-x_{3}, \quad c_{3}=x_{2}-x_{1}
$$

are related to the nodal coordinates, $\left(\Delta_{1}, \Delta_{2}, \Delta_{3}\right)$ are equal to the areas of triangles P23, P31 and P12, respectively, as shown in sketch figure of Fig. $1 ; \Delta$ is area of triangle 123; $\left(x_{i}, y_{i}\right)(i=1,2,3)$ are the coordinates of nodes 1,2 and 3.

For simplicity, firstly, Eqs. (15), (16) and (17) are expressed into the matrix forms:

$$
\begin{aligned}
& \int_{V}\left\{\delta \boldsymbol{U}^{\mathrm{T}} \cdot \boldsymbol{\sigma}+\boldsymbol{\delta}(\boldsymbol{\nabla} \boldsymbol{U})^{\mathrm{T}} \cdot \boldsymbol{\tau}\right\} \mathrm{d} V=\int_{V} \delta \boldsymbol{U}^{\mathrm{T}} \cdot \boldsymbol{f}^{\mathrm{B}} \mathrm{d} V+\int_{S} \delta \boldsymbol{U}^{\mathrm{T}} \cdot M \mathrm{~d} S, \\
& \boldsymbol{\sigma}=\boldsymbol{D} \cdot \boldsymbol{U}, \quad \boldsymbol{\tau}=\boldsymbol{C} \cdot(\boldsymbol{\nabla} \boldsymbol{U}) .
\end{aligned}
$$

Stiffness modulus matrices $\boldsymbol{D}$ and $\boldsymbol{C}$ can be easily formulated from constitutive relations (10). Displacement gradient $\boldsymbol{U}$ can be expressed by its node value $\boldsymbol{U}^{\mathrm{e}}$ in each element,

$$
\boldsymbol{U}=\boldsymbol{N} \cdot \boldsymbol{U}^{\mathrm{e}}
$$

then

$$
(\nabla \boldsymbol{U})=\boldsymbol{B} \cdot \boldsymbol{U}^{\mathrm{e}}, \quad \boldsymbol{B}=\nabla N,
$$

where $N$ is shape function matrix; $\boldsymbol{B}$ is strain matrix. Substitute (24), (25) and (26) into (23), for each element, we have

$$
\int_{V^{\mathrm{e}}}\left\{\boldsymbol{N}^{\mathrm{T}} \boldsymbol{D} \boldsymbol{N}+\boldsymbol{B}^{\mathrm{T}} \boldsymbol{C} \boldsymbol{B}\right\} \mathrm{d} V \cdot \boldsymbol{U}^{\mathrm{e}}=\int_{V^{\mathrm{e}}} \boldsymbol{N}^{\mathrm{T}} \boldsymbol{f}^{\mathrm{B}} \mathrm{d} V+\int_{S^{\mathrm{e}}} \boldsymbol{N}^{\mathrm{T}} \boldsymbol{M} \mathrm{d} S,
$$



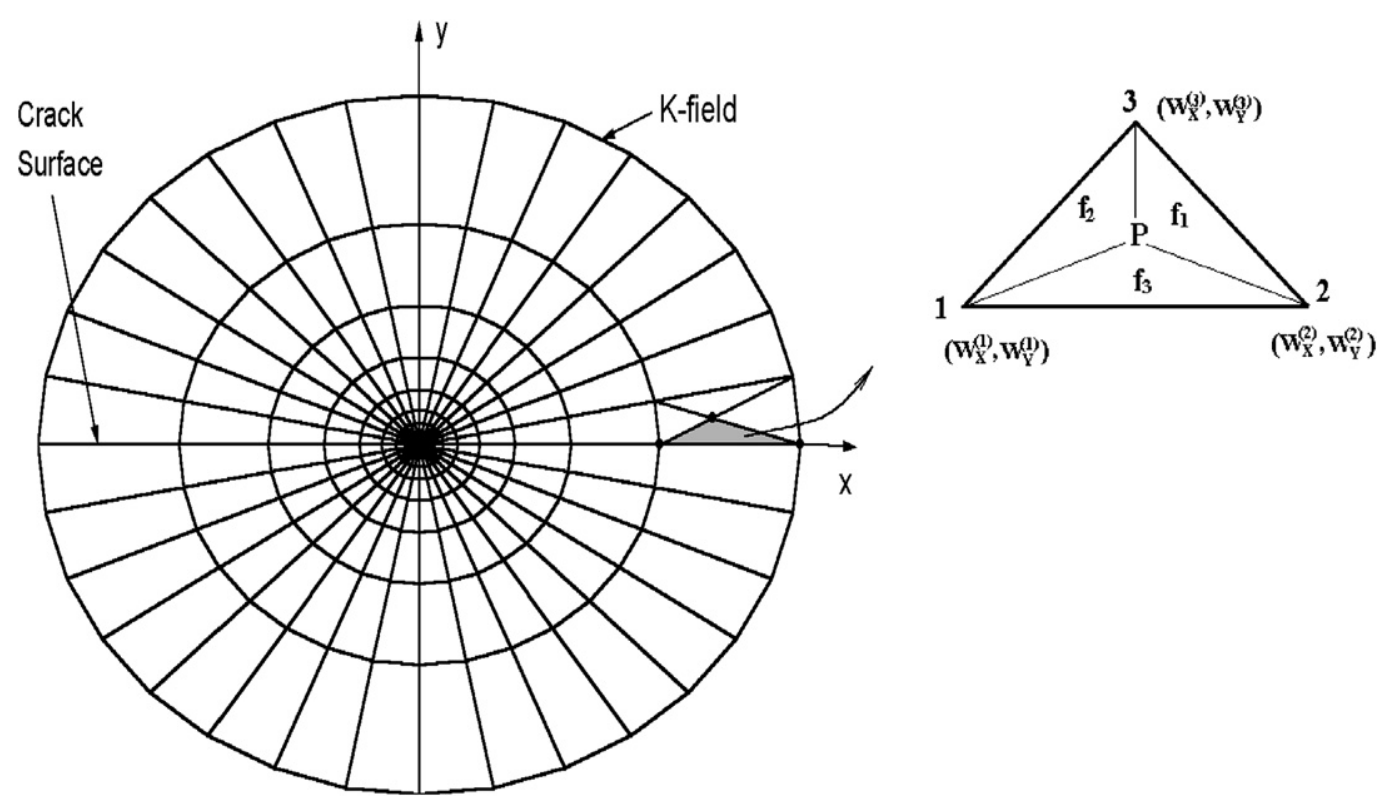

Fig. 1. Finite element mesh and triangle element and the area coordinates. Nodal variables are displacement derivatives.

where superscription "e" stands for an element. $V^{\mathrm{e}}$ and $\boldsymbol{S}^{\mathrm{e}}$ are the element volume and boundary surface. Frequently (27) is simply expressed in the form:

$$
\boldsymbol{K}^{\mathrm{e}} \cdot \boldsymbol{U}^{\mathrm{e}}=\boldsymbol{F}^{\mathrm{e}}
$$

where

$$
\boldsymbol{K}^{\mathrm{e}}=\int_{V^{\mathrm{e}}}\left\{\boldsymbol{N}^{\mathrm{T}} \boldsymbol{D} \boldsymbol{N}+\boldsymbol{B}^{\mathrm{T}} \boldsymbol{C} \boldsymbol{B}\right\} \mathrm{d} V, \quad \boldsymbol{F}^{\mathrm{e}}=\int_{V^{\mathrm{e}}} \boldsymbol{N}^{\mathrm{T}} \boldsymbol{f}^{\mathrm{B}} \mathrm{d} V+\int_{S^{\mathrm{e}}} \boldsymbol{N}^{\mathrm{T}} \boldsymbol{M} \mathrm{d} S
$$

are the element stiffness matrix and element node force matrix, respectively. Assembling all element relations by using (23) and (27), the global stiffness equation is obtained as:

$$
\boldsymbol{K} \cdot \boldsymbol{U}=\boldsymbol{F},
$$

where

$$
\boldsymbol{K}=\sum_{\mathrm{e}} \int_{V^{\mathrm{e}}}\left\{\boldsymbol{N}^{\mathrm{T}} \boldsymbol{D} \boldsymbol{N}+\boldsymbol{B}^{\mathrm{T}} \boldsymbol{C} \boldsymbol{B}\right\} \mathrm{d} V, \quad \boldsymbol{F}=\sum_{\mathrm{e}}\left\{\int_{V^{\mathrm{e}}} \boldsymbol{N}^{\mathrm{T}} \boldsymbol{f}^{\mathrm{B}} \mathrm{d} V+\int_{S^{\mathrm{e}}} \boldsymbol{N}^{\mathrm{T}} \boldsymbol{M} \mathrm{d} S\right\}
$$

are the globe stiffness matrix and node force matrix, respectively. Relations (25)-(31) show the outlines of the new finite element method. In order to put the new finite element method into application, it is important to further study the forms of the shape function matrix and node variable matrix. For simplicity, consider a simple case first, where the single displacement component $w$ is concerned. The case of multi-displacement components (such as $u, v$, etc.) can be generalized simply. The displacement derivatives for the single displacement $w$ are calculated by

$$
U_{i j} \equiv U_{3 j}=(\partial w / \partial x, \partial w / \partial y)=\left(w_{x}, w_{y}\right) .
$$

The displacement gradient components can be expressed by using the nodal variables as

$$
w_{x}=\sum_{I=1}^{3}\left\{N_{1}^{(I)} W_{X}^{(I)}+N_{2}^{(I)} W_{Y}^{(I)}\right\}, \quad w_{y}=\sum_{I=1}^{3}\left\{\bar{N}_{1}^{(I)} W_{X}^{(I)}+\bar{N}_{2}^{(I)} W_{Y}^{(I)}\right\},
$$

where $\left(W_{X}^{(I)}, W_{Y}^{(I)}\right)(I=1,2,3)$ are the node variables of element; the shape functions $\left(N_{1}^{(I)}, N_{2}^{(I)}\right)$ and $\left(\bar{N}_{1}^{(I)}, \bar{N}_{2}^{(I)}\right)$ $(I=1,2,3)$ will be determined by selecting a polynomial functions for $\left(w_{x}, w_{y}\right)$ according to six continuous 
conditions at all three nodes and one compatible condition $w_{x y}=w_{y x}$. Consider a cubic polynomial relation for displacement function

$$
w(x, y)=A_{0}+A_{1} x+A_{2} y+A_{3} f_{1} f_{2}+A_{4} f_{2} f_{3}+A_{5} f_{3} f_{1}+A_{6}\left(f_{1}^{2} f_{2}+f_{2}^{2} f_{3}+f_{3}^{2} f_{1}\right) .
$$

Thus the compatible condition, $w_{x y}=w_{y x}$, can be satisfied automatically. From (33), we have

$$
\begin{aligned}
w_{x}= & A_{1}+\left\{A_{3}\left(b_{1} f_{2}+b_{2} f_{1}\right)+A_{4}\left(b_{2} f_{3}+b_{3} f_{2}\right)+A_{5}\left(b_{3} f_{1}+b_{1} f_{3}\right)+A_{6}\left(b_{2} f_{1}^{2}+b_{3} f_{2}^{2}+b_{1} f_{3}^{2}\right.\right. \\
& \left.\left.+2 b_{1} f_{1} f_{2}+2 b_{2} f_{2} f_{3}+2 b_{3} f_{3} f_{1}\right)\right\} / \Delta \\
w_{y}= & A_{2}+\left\{A_{3}\left(c_{1} f_{2}+c_{2} f_{1}\right)+A_{4}\left(c_{2} f_{3}+c_{3} f_{2}\right)+A_{5}\left(c_{3} f_{1}+c_{1} f_{3}\right)+A_{6}\left(c_{2} f_{1}^{2}+c_{3} f_{2}^{2}+c_{1} f_{3}^{2}\right.\right. \\
& \left.\left.+2 c_{1} f_{1} f_{2}+2 c_{2} f_{2} f_{3}+2 c_{3} f_{3} f_{1}\right)\right\} / \Delta,
\end{aligned}
$$

where $b_{i}, c_{i}(i=1,2,3)$ have been given in (22), $A_{i}(i=1,2, \ldots, 6)$ are constants to be determined from continuity conditions of $\left(w_{x}, w_{y}\right)$ at nodes, and can be expressed by the node variables according to the conditions: $\left.\left(w_{x}, w_{y}\right)\right|_{(I)}=\left(W_{X}^{(I)}, W_{Y}^{(I)}\right)(I=1,2,3) . A_{0}$ can be taken as the element rigid displacement, and it can be determined after the solution $\left(w_{x}, w_{y}\right)$ is found if one wants to find displacement field. Through a longer derivation and simplification, we obtain

$$
\begin{aligned}
& A_{1}=\frac{1}{3}\left\{W_{X}^{(1)}+W_{X}^{(2)}+W_{X}^{(3)}+A_{3} b_{3} / \Delta+A_{4} b_{1} / \Delta+A_{5} b_{2} / \Delta\right\}, \\
& A_{2}=\frac{1}{3}\left\{W_{Y}^{(1)}+W_{Y}^{(2)}+W_{Y}^{(3)}+A_{3} c_{3} / \Delta+A_{4} c_{1} / \Delta+A_{5} c_{2} / \Delta\right\}, \\
& A_{3}=\frac{1}{2} c_{3}\left(W_{X}^{(1)}-W_{X}^{(2)}\right)-\frac{1}{2} b_{3}\left(W_{Y}^{(1)}-W_{Y}^{(2)}\right)-\frac{1}{2} A_{6}, \\
& A_{4}=\frac{1}{2} c_{1}\left(W_{X}^{(2)}-W_{X}^{(3)}\right)-\frac{1}{2} b_{1}\left(W_{Y}^{(2)}-W_{Y}^{(3)}\right)-\frac{1}{2} A_{6}, \\
& A_{5}=\frac{1}{2} c_{2}\left(W_{X}^{(3)}-W_{X}^{(1)}\right)-\frac{1}{2} b_{2}\left(W_{Y}^{(3)}-W_{Y}^{(1)}\right)-\frac{1}{2} A_{6}, \\
& A_{6}=\frac{1}{3}\left\{-c_{1} W_{X}^{(1)}-c_{2} W_{X}^{(2)}-c_{3} W_{X}^{(3)}+b_{1} W_{Y}^{(1)}+b_{2} W_{Y}^{(2)}+b_{3} W_{Y}^{(3)}\right\} .
\end{aligned}
$$

From (32), (34) and (35), the expressions of shape functions for node $i$ can be derived out:

$$
\begin{aligned}
N_{1}^{(i)}= & \frac{1}{3}-\frac{1}{2} c_{j}\left(\frac{1}{3} b_{j}+b_{k} f_{i}+b_{i} f_{k}\right) / \Delta+\frac{1}{2} c_{k}\left(\frac{1}{3} b_{k}+b_{i} f_{j}+b_{j} f_{i}\right) / \Delta \\
& -\frac{1}{3} c_{i}\left\{\frac{1}{2}\left(b_{i} f_{i}+b_{j} f_{j}+b_{k} f_{k}\right)+f_{i}^{2} b_{j}+f_{j}^{2} b_{k}+f_{k}^{2} b_{i}+2\left(b_{i} f_{i} f_{j}+b_{j} f_{j} f_{k}+b_{k} f_{k} f_{i}\right)\right\} / \Delta, \\
N_{2}^{(i)}= & \frac{1}{2} b_{j}\left(\frac{1}{3} b_{j}+b_{k} f_{i}+b_{i} f_{k}\right) / \Delta-\frac{1}{2} b_{k}\left(\frac{1}{3} b_{k}+b_{i} f_{j}+b_{j} f_{i}\right) / \Delta \\
& +\frac{1}{3} b_{i}\left\{\frac{1}{2}\left(b_{i} f_{i}+b_{j} f_{j}+b_{k} f_{k}\right)+f_{i}^{2} b_{j}+f_{j}^{2} b_{k}+f_{k}^{2} b_{i}+2\left(b_{i} f_{i} f_{j}+b_{j} f_{j} f_{k}+b_{k} f_{k} f_{i}\right)\right\} / \Delta, \\
\bar{N}_{1}^{(i)}= & -\frac{1}{2} c_{j}\left(\frac{1}{3} c_{j}+c_{k} f_{i}+c_{i} f_{k}\right) / \Delta+\frac{1}{2} c_{k}\left(\frac{1}{3} c_{k}+c_{i} f_{j}+c_{j} f_{i}\right) / \Delta \\
& -\frac{1}{3} c_{i}\left\{\frac{1}{2}\left(c_{i} f_{i}+c_{j} f_{j}+c_{k} f_{k}\right)+f_{i}^{2} c_{j}+f_{j}^{2} c_{k}+f_{k}^{2} c_{i}+2\left(c_{i} f_{i} f_{j}+c_{j} f_{j} f_{k}+c_{k} f_{k} f_{i}\right)\right\} / \Delta, \\
\bar{N}_{2}^{(i)}= & \frac{1}{3}+\frac{1}{2} b_{j}\left(\frac{1}{3} c_{j}+c_{k} f_{i}+c_{i} f_{k}\right) / \Delta-\frac{1}{2} b_{k}\left(\frac{1}{3} c_{k}+c_{i} f_{j}+c_{j} f_{i}\right) / \Delta \\
& +\frac{1}{3} b_{i}\left\{\frac{1}{2}\left(c_{i} f_{i}+c_{j} f_{j}+c_{k} f_{k}\right)+f_{i}^{2} c_{j}+f_{j}^{2} c_{k}+f_{k}^{2} c_{i}+2\left(c_{i} f_{i} f_{j}+c_{j} f_{j} f_{k}+c_{k} f_{k} f_{i}\right)\right\} / \Delta .
\end{aligned}
$$

$(i, j, k)$ are the cyclic permutations of $1,2,3$. The new finite element method can be used to calculate the strain gradient problems by substituting (36) into (26), (30) and (31). 
For comparison, the fundamental relation of the Zienkiewicz-Taylor $\mathrm{C} 1$ finite element method is discussed briefly. The variational relation (18) can be directly transferred to the finite element formulations. The form of the variational relation (18) implies that both the displacements and displacement gradients can be taken as the fundamental variables. This can be directly connected with the Zienkiewicz-Taylor C1 finite element scheme (Zienkiewicz and Taylor, 1989a, 1989b). A detail discussion was given in Xia and Hutchinson (1996). The fundamental formulations of the C1 finite element scheme were derived by Zienkiewicz and Taylor (1989a, 1989b) and by Xia and Hutchinson (1996). For a triangle element, the shape function for nodal variables of both displacements and displacement derivatives was given as follows (from Xia and Hutchinson, 1996)

$$
\boldsymbol{N}_{i}^{\mathrm{T}}=\left(\begin{array}{c}
3 f_{i}^{2}-2 f_{i}^{3}-2 f_{i} f_{j} f_{k}, \\
-c_{j}\left(f_{i}^{2} f_{k}+f_{i} f_{j} f_{k}\right)+c_{k} f_{i}^{2} f_{j}, \\
b_{j}\left(f_{i}^{2} f_{k}+f_{i} f_{j} f_{k}\right)-b_{k} f_{i}^{2} f_{j}
\end{array}\right),
$$

$(i, j, k)$ are the cyclic permutations of 1,2,3 for each nodes. Three expressions in (37) correspond to nodal variables $\left(w, w_{x}, w_{y}\right)$ at node $i$. The $\mathrm{C} 1$ finite element method is referred to the continuity of both the displacement and displacement derivative across the element boundary nodes. Based on (10) and (18), the corresponding globe stiffness matrix and the node force matrix can be derived similarly with the derivations of Eqs. (30) and (31) for the new finite element method. However, the fundamental variables include both displacements and their derivatives, as described by Xia and Hutchinson (1996). The effectiveness of the $\mathrm{C} 1$ finite element method in application to the strain gradient problems will be discussed in Section 6.

\section{Matrix expressions of some fundamental problems for new finite element method}

In order to present the applications of the new finite element method, let us further discuss the matrix expressions in detail for some typical cases, such as the anti-plane shear and the plane strain.

\subsection{Anti-plane shear}

For the anti-plane shear case, the fundamental variables in matrix forms can be dictated as follows

$$
\begin{aligned}
& \boldsymbol{U}=\left(\begin{array}{l}
w_{x} \\
w_{y}
\end{array}\right)=\left(\begin{array}{l}
\gamma_{31} \\
\gamma_{32}
\end{array}\right), \quad \nabla \boldsymbol{U}=\left(\begin{array}{c}
w_{x x} \\
w_{y y} \\
2 w_{x y}
\end{array}\right)=\left(\begin{array}{c}
\eta_{113} \\
\eta_{223} \\
2 \eta_{213}
\end{array}\right), \quad \boldsymbol{\sigma}=\left(\begin{array}{c}
\sigma_{13} \\
\sigma_{23}
\end{array}\right), \quad \boldsymbol{\tau}=\left(\begin{array}{c}
\tau_{113} \\
\tau_{223} \\
\tau_{213}
\end{array}\right), \\
& \boldsymbol{f}^{\mathrm{B}}=\left(\begin{array}{l}
\sigma_{13}-\tau_{113,1}-\tau_{213,2} \\
\sigma_{23}-\tau_{123,1}-\tau_{223,2}
\end{array}\right), \quad \boldsymbol{M}=\left(\begin{array}{l}
n_{1} \tau_{113}+n_{2} \tau_{213} \\
n_{1} \tau_{123}+n_{2} \tau_{223}
\end{array}\right),
\end{aligned}
$$

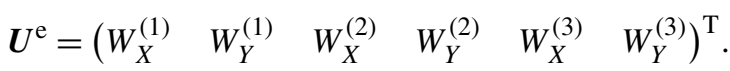

In (38), $\tau_{123}=\tau_{213}$ is considered, and some non-zero components of higher-order stresses are not listed, because they will not appear in the variation equation (23), however in calculating the effective stress the omitted terms will be included. In (38), $n_{i}$ is the direction cosine of $S$. From (32), (38) and (39), the corresponding shape function and strain matrices become

$$
\begin{aligned}
\boldsymbol{N} & =\left(\begin{array}{llllll}
N_{1}^{(1)} & N_{2}^{(1)} & N_{1}^{(2)} & N_{2}^{(2)} & N_{1}^{(3)} & N_{2}^{(3)} \\
\bar{N}_{1}^{(1)} & \bar{N}_{2}^{(1)} & \bar{N}_{1}^{(2)} & \bar{N}_{2}^{(2)} & \bar{N}_{1}^{(3)} & \bar{N}_{2}^{(3)}
\end{array}\right), \\
\boldsymbol{B} & =\left(\begin{array}{cccccc}
N_{1 x}^{(1)} & N_{2 x}^{(1)} & N_{1 x}^{(2)} & N_{2 x}^{(2)} & N_{1 x}^{(3)} & N_{2 x}^{(3)} \\
\bar{N}_{1 y}^{(1)} & \bar{N}_{2 y}^{(1)} & \bar{N}_{1 y}^{(2)} & \bar{N}_{2 y}^{(2)} & \bar{N}_{1 y}^{(3)} & \bar{N}_{2 y}^{(3)} \\
2 N_{1 y}^{(1)} & 2 N_{2 y}^{(1)} & 2 N_{1 y}^{(2)} & 2 N_{2 y}^{(2)} & 2 N_{1 y}^{(3)} & 2 N_{2 y}^{(3)}
\end{array}\right) .
\end{aligned}
$$

\subsection{Plane strain}

For plane strain case, fundamental displacement components are $(u, v)$. The shape functions of displacement gradient components can be taken as the same form as that for $w$ in above anti-plane shear case (see (33)). The matrix forms of the fundamental variables are given as follows 


$$
\begin{aligned}
& \boldsymbol{U}=\left(\begin{array}{llll}
u_{x} & v_{y} & u_{y} & v_{x}
\end{array}\right)^{\mathrm{T}}, \\
& \nabla \boldsymbol{U}=\left(\begin{array}{llllllllll}
u_{x x} & u_{y y} & 2 v_{x y} & v_{y y} & v_{x x} & 2 u_{x y}
\end{array}\right)^{\mathrm{T}}=\left(\begin{array}{llllll}
\eta_{111} & \eta_{221} & 2 \eta_{122} & \eta_{222} & \eta_{112} & 2 \eta_{121}
\end{array}\right)^{\mathrm{T}}, \\
& \boldsymbol{\sigma}=\left(\begin{array}{llll}
\sigma_{11} & \sigma_{22} & \sigma_{12} & \sigma_{21}
\end{array}\right)^{\mathrm{T}}, \quad \boldsymbol{\tau}=\left(\begin{array}{llllll}
\tau_{111} & \tau_{221} & \tau_{122} & \tau_{222} & \tau_{112} & \tau_{121}
\end{array}\right)^{\mathrm{T}}, \\
& \boldsymbol{f}^{\mathrm{B}}=\left(\begin{array}{l}
\sigma_{11}-\tau_{111,1}-\tau_{211,2} \\
\sigma_{22}-\tau_{122,1}-\tau_{222,2} \\
\sigma_{12}-\tau_{121,1}-\tau_{221,2} \\
\sigma_{21}-\tau_{112,1}-\tau_{212,2}
\end{array}\right), \quad \boldsymbol{M}=\left(\begin{array}{l}
n_{1} \tau_{111}+n_{2} \tau_{211} \\
n_{1} \tau_{122}+n_{2} \tau_{222} \\
n_{1} \tau_{121}+n_{2} \tau_{221} \\
n_{1} \tau_{112}+n_{2} \tau_{212}
\end{array}\right), \\
& \boldsymbol{U}^{\mathrm{e}}=\left(\begin{array}{llllllllllll}
U_{X}^{(1)} & V_{X}^{(1)} & U_{Y}^{(1)} & V_{Y}^{(1)} & U_{X}^{(2)} & V_{X}^{(2)} & U_{Y}^{(2)} & V_{Y}^{(2)} & U_{X}^{(3)} & V_{X}^{(3)} & U_{Y}^{(3)} & V_{Y}^{(3)}
\end{array}\right)^{\mathrm{T}} .
\end{aligned}
$$

In (42), some non-zero higher-order stress components are not listed there, because they do not appear in the variational equation (23). The shape function matrix and strain matrix can be expressed as

$$
\begin{aligned}
& \boldsymbol{N}=\left(\begin{array}{cccccccccccc}
N_{1}^{(1)} & 0 & N_{2}^{(1)} & 0 & N_{1}^{(2)} & 0 & N_{2}^{(2)} & 0 & N_{1}^{(3)} & 0 & N_{2}^{(3)} & 0 \\
0 & \bar{N}_{1}^{(1)} & 0 & \bar{N}_{2}^{(1)} & 0 & \bar{N}_{1}^{(2)} & 0 & \bar{N}_{2}^{(2)} & 0 & \bar{N}_{1}^{(3)} & 0 & \bar{N}_{2}^{(3)} \\
\bar{N}_{1}^{(1)} & 0 & \bar{N}_{2}^{(1)} & 0 & \bar{N}_{1}^{(2)} & 0 & \bar{N}_{2}^{(2)} & 0 & \bar{N}_{1}^{(3)} & 0 & \bar{N}_{2}^{(3)} & 0 \\
0 & N_{1}^{(1)} & 0 & N_{2}^{(1)} & 0 & N_{1}^{(2)} & 0 & N_{2}^{(2)} & 0 & N_{1}^{(3)} & 0 & N_{2}^{(3)}
\end{array}\right), \\
& \boldsymbol{B}=\left(\begin{array}{cccccccccccc}
N_{1 x}^{(1)} & 0 & N_{2 x}^{(1)} & 0 & N_{1 x}^{(2)} & 0 & N_{2 x}^{(2)} & 0 & N_{1 x}^{(3)} & 0 & N_{2 x}^{(3)} & 0 \\
\bar{N}_{1 y}^{(1)} & 0 & \bar{N}_{2 y}^{(1)} & 0 & \bar{N}_{1 y}^{(2)} & 0 & \bar{N}_{2 y}^{(2)} & 0 & \bar{N}_{1 y}^{(3)} & 0 & \bar{N}_{2 y}^{(3)} & 0 \\
0 & 2 N_{1 y}^{(1)} & 0 & 2 N_{2 y}^{(1)} & 0 & 2 N_{1 y}^{(2)} & 0 & 2 N_{2 y}^{(2)} & 0 & 2 N_{1 y}^{(3)} & 0 & 2 N_{2 y}^{(3)} \\
0 & \bar{N}_{1 y}^{(1)} & 0 & \bar{N}_{2 y}^{(1)} & 0 & \bar{N}_{1 y}^{(2)} & 0 & \bar{N}_{2 y}^{(2)} & 0 & \bar{N}_{1 y}^{(3)} & 0 & \bar{N}_{2 y}^{(3)} \\
0 & N_{1 x}^{(1)} & 0 & N_{2 x}^{(1)} & 0 & N_{1 x}^{(2)} & 0 & N_{2 x}^{(2)} & 0 & N_{1 x}^{(3)} & 0 & N_{2 x}^{(3)} \\
2 N_{1 y}^{(1)} & 0 & 2 N_{2 y}^{(1)} & 0 & 2 N_{1 y}^{(2)} & 0 & 2 N_{2 y}^{(2)} & 0 & 2 N_{1 y}^{(3)} & 0 & 2 N_{2 y}^{(3)} & 0
\end{array}\right) .
\end{aligned}
$$

\section{Solution procedures using the new finite element method}

In the fundamental relations of the new finite element method, stiffness modulus $\boldsymbol{D}$ and $\boldsymbol{C}$ in (31) can be written easily through comparing (10) (or (11)) and (24), and the equivalent node force matrix $\boldsymbol{F}$ can be calculated with the equivalent body force $\boldsymbol{f}^{\mathrm{B}}$ and the surface toque $\boldsymbol{M} \cdot \boldsymbol{f}^{\mathrm{B}}$ and $\boldsymbol{M}$ can be calculated using formulas (38) or (42) for the anti-plane shear case or plane strain case. The steps of solving a strain gradient problem using the new finite element method can be dictated as follows:

(1) Take the corresponding conventional elastic stress solution as the initial equivalent body force (matrix $f^{\mathrm{B}}$ ), and let the initial surface toque matrix $\boldsymbol{M}$ be zero. Solve Eq. (30), and get the first trial solution;

(2) Substitute the solution of the first step into (38) or (42) to calculate the new $\boldsymbol{f}^{\mathrm{B}}$ and $\boldsymbol{M}$. Then substitute them into (31) to calculate $\boldsymbol{K}$ and $\boldsymbol{F}$, and then solve the matrix equation (30);

(3) Use the new solution to calculate the new $\boldsymbol{f}^{\mathrm{B}}$ and $\boldsymbol{M}$. Repeat the iteration procedure mentioned above until a convergent solution is obtained.

For the crack problems, the conventional K-stress fields are used to calculate the initial equivalent body forces.

In the present study, our attention will be mainly focused on the investigation of the traction distributions along the plane ahead of the crack tip within the microscale region. Traction formula considering strain gradient effects can be given from (19) for $\left(n_{1}, n_{2}\right)=(0,1)$,

$$
t_{2 k}=\sigma_{2 k}-2 \frac{\partial \tau_{21 k}}{\partial x_{1}}-\frac{\partial \tau_{22 k}}{\partial x_{2}} \text {. }
$$

Mode I, Mode II and Mode III fracture problems correspond to $k=2, k=1$ and $k=3$, respectively. 


\section{Effectiveness testing of the new finite element methods}

The effectiveness of the new finite element method can be assessed by applying it to the analyses for some typical problems which have had the closed-form analytical solutions, and through comparing the numerical solutions with the corresponding exact solutions.

It is worth pointing out that the most successful application of the new finite element method is to the BernoulliEuler beam bending problems, the exact solution is always obtained by using the new finite element method, no matter how dense the element divided. The reason is that both exact solution of the Bernoulli-Euler beam bending problems and the new finite element method solution are polynomial forms.

In this section, we focus attention to that the new finite element methods developed above will be used to analyze the typical fracture problems in strain gradient elasticity, about which Huang and his collaborators have obtained the closed-form (exact) solutions. For comparison, in the analyses for the mode III fracture problem, the ZienkiewiczTaylor $\mathrm{C} 1$ finite element method is also adopted. The finite element mesh is shown in Fig. 1. The basic element is the triangle element, which is formed by dividing a quadrilateral into four parts, as shown in Fig. 1. The boundary conditions include two parts, i.e., the remote boundary condition and crack surface boundary condition. At the remote boundary, K-field is exerted. On the crack surfaces, traction-free and toque-free boundary conditions are exerted.

\subsection{Anti-plane shear-Mode III fracture}

Zhang et al. (1998) and Shi et al. (2000) have obtained the closed form solutions for fundamental fracture problem (Mode III) in strain gradient elasticity, based on the Wiener-Hopf techniques. The closed-form (exact) solution, normal traction distribution on the plane ahead of the crack tip, will be served as the main benchmark for the numerical methods.

Firstly, the Zienkiewicz-Taylor C1 finite element method is used to calculate the traction distribution. In our calculation, the second selection of the specimen point proposed in Specht (1988) is adopted. The result is shown in Fig. 2. Simultaneously, the solutions obtained by Huang and his collaborators, the asymptotic solution, closed-form (exact) solution, as well as the classical $\mathrm{K}_{\mathrm{III}}-$ field, are also shown in the figure. Through comparison, surprisingly, the Zienkiewicz-Taylor $\mathrm{C} 1$ finite element solution fits close to the asymptotic solution, and is far away from the exact solution. Note that asymptotic solution estimates only the role of the higher-order singularity-dominated terms, i.e., the role of the higher-order stress and strain terms in strain gradient theory. Therefore, the result of the ZienkiewiczTaylor $\mathrm{C} 1$ finite element method shown in Fig. 2 implies that the role of the terms of conventional stresses and strains in the strain gradient theory is underestimated or submerged. This leads us to check the effectiveness of the Zienkiewicz-Taylor $\mathrm{C} 1$ finite element method in being applied to the strain gradient problems. Why could one obtain an effective solution for a plate-bending problem by using the $\mathrm{C} 1$ finite element method (Zienkiewicz and Taylor, 1989a, 1989b), and why cannot obtain the effective solution for the strain gradient problem? Through comparison, we observe that there exist some differences between the plate-bending theory and the strain gradient theory. For the plate-bending theory there only exist the terms of the second-order derivatives of displacements (curvatures), i.e., pure "strain gradient" terms, and not include the displacement gradient terms in the constitutive relations. However, for the strain gradient theory, both the strain gradient terms and the displacement gradient terms are all included in the constitutive relations. According to fundamental requirement for an effective finite element method, for the plate bending theory the finite element method should characterize a constant curvature state effectively ("constant strain condition"), and the $\mathrm{C} 1$ finite element method satisfies the condition. However, applying the $\mathrm{C} 1$ finite element method to the problems described by the strain gradient theory, the "constant strain condition" is not clear, because we have both the higher-order strain terms and the conventional strain terms simultaneously, and these immediately lead to two different "constant strain conditions". Through further investigating, we conclude that the two different constant strain requirements are difficult or even impossible to be satisfied simultaneously. From the result shown in Fig. 1 using the C1 finite element method, likely, the constant strain condition for the higher-order strain seems to be satisfied, while the constant strain condition for conventional strain terms seems not to be satisfied.

In order to find the solution for mode III strain gradient elasticity problem by using the new finite element method, in the first step, we take the conventional $\mathrm{K}_{\mathrm{III}}$-stress field as the initial equivalent body force and set the initial surface toque to be zero, $\boldsymbol{M}=\mathbf{0}$. Calculate the equivalent node force from (31), and solve the finite element equation (30) to get the first trial solution. Use the trial solution to calculate the new equivalent body force and surface toque and 


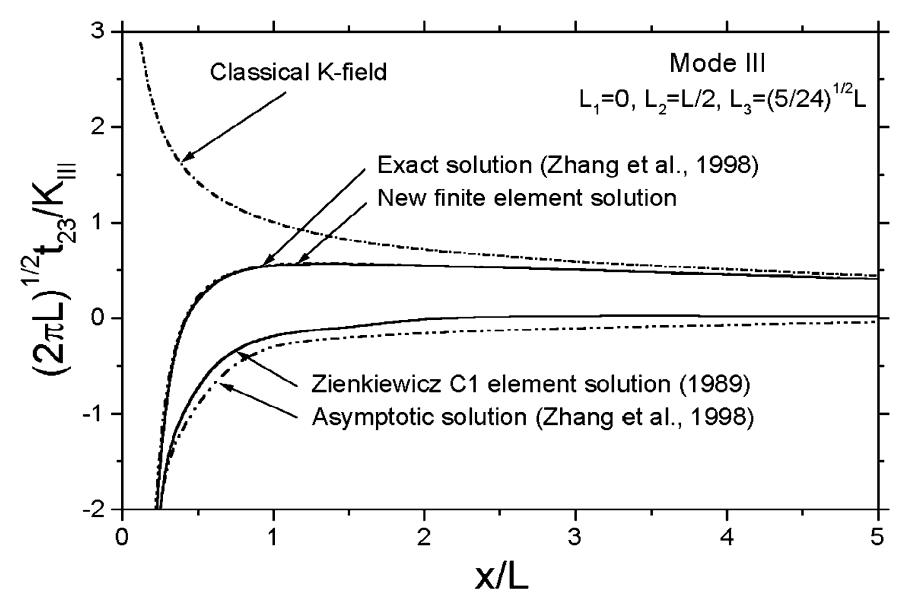

Fig. 2. Comparison of the new finite element method result and the Zienkiewicz-Taylor C1 element result with exact solution for Mode III fracture.

further solve (30) iteratively, until a convergent solution is obtained. In our calculations, take the central point of triangle element as the specimen point (we also adopted the four selections of the specimen points proposed in Specht (1988), and obtained near same solutions). After a convergent solution is obtained, the traction distribution on the plane ahead of the crack tip is calculated by using (46).

The comparison of the new finite element solution with the closed-form solution by Zhang et al. (1998) is shown in Fig. 2. The exact solution is based on the couple stress theory (Fleck and Hutchinson, 1993), which corresponds to a special selection for the length parameter group $L_{I}$ in general strain gradient plasticity theory (Fleck and Hutchinson, 1997), see formula (13). From Fig. 2, the solution of traction distribution using new finite element method is very consistent with the exact solution. In the new finite element method, since the conventional stress terms are treated as the body force, the higher-order strain and stress terms are only concerned on the analysis process. Therefore, the "constant strain condition" is referred to the higher-order strain terms, and obviously can be satisfied.

\subsection{In-plane shear - Mode II fracture}

Consider Mode II plane strain fracture problem in strain gradient elasticity. Similarly, analysis using the new finite element method starts from selecting the conventional linear fracture stress field (classical $\mathrm{K}_{\mathrm{II}}$-field) as the initial equivalent body force and selecting zero-initial toque. Solve Eq. (30) iteratively. Using formula (46), the traction distribution on the plane ahead of the crack tip is calculated. The result is shown in Fig. 3. Fig. 3 (a) and (b) show the results for two group selections of parameters $L_{I}(I=1,3)$, previously taken in Shi et al. (2000). When $L_{1}$ is equal to zero, corresponds to the couple stress case, see (13). As $L_{1}$ increases, the stretch effect of the strain gradients increases. The curve of the exact solution given by Shi et al. (2000) is also plotted. Obviously, the new finite element solution is very consistent with the exact solution.

\subsection{Plane strain-Mode I fracture}

For Mode I fracture problem in the strain gradient elasticity, Shi et al. (2000) have solved the case and obtained a closed-form (exact) solution when material obeys the incompressible condition, which corresponds to that let the material Poisson ratio be equal to 0.5. We start our analysis from the general compressible case, since when Poisson ratio is exactly set to 0.5 , the modulus matrix in finite element method will become singular from the expressions of matrices $\boldsymbol{C}$ and $\boldsymbol{D}$ referring to (24), (29), (10) and (11). In view of the reason, we consider a series of values of Poisson's ratio from 0.3 to 0.495 to approach an incompressible state and compare the solution with the incompressible exact solution of Shi et al. (2000) for further checking the effectiveness of the new finite element method.

Similarly, the iterative solution procedures are needed in solving the Mode I fracture problem. Take the conventional linear fracture $\mathrm{K}_{\mathrm{I}}$-stress field as the initial equivalent body force and set $\boldsymbol{M}=\mathbf{0}$. The new finite element solutions are given in Fig. 4 for traction distribution along the plane ahead of the crack tip calculated by using (46). The curves shown in figure correspond to several values of Poisson's ratio from 0.3 to 0.495 . From the solution, when material 


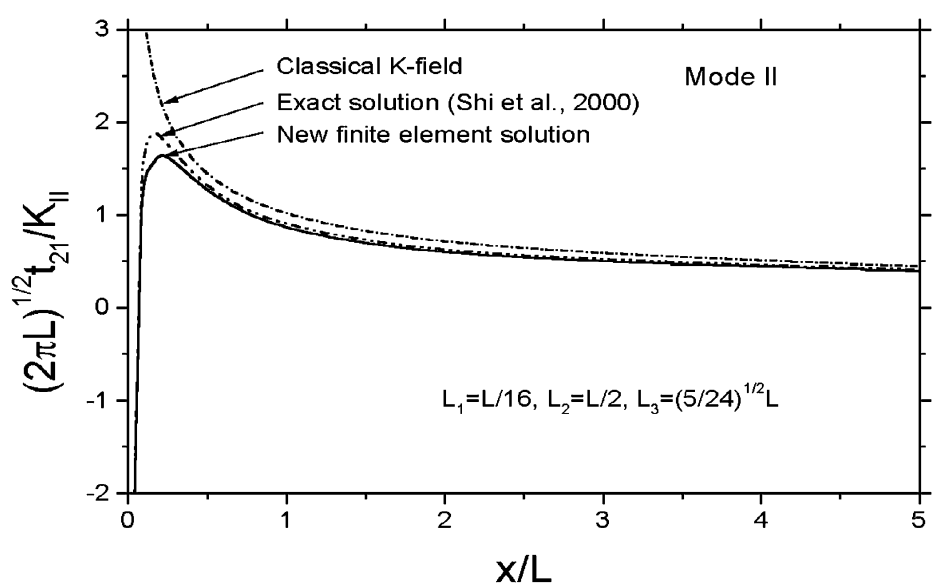

(a)

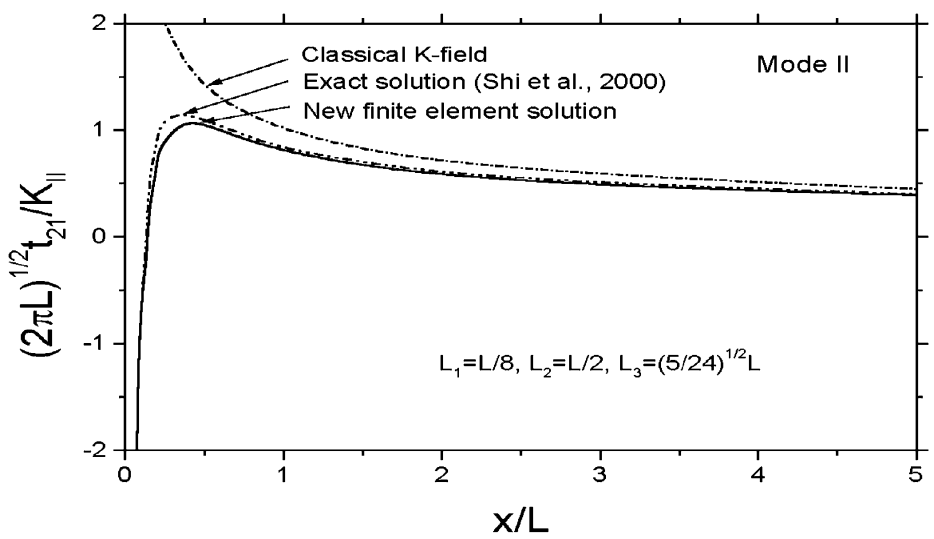

(b)

Fig. 3. Comparison of new finite element method result with exact solution for Mode II fracture. (a) and (b) for different length parameter selections.

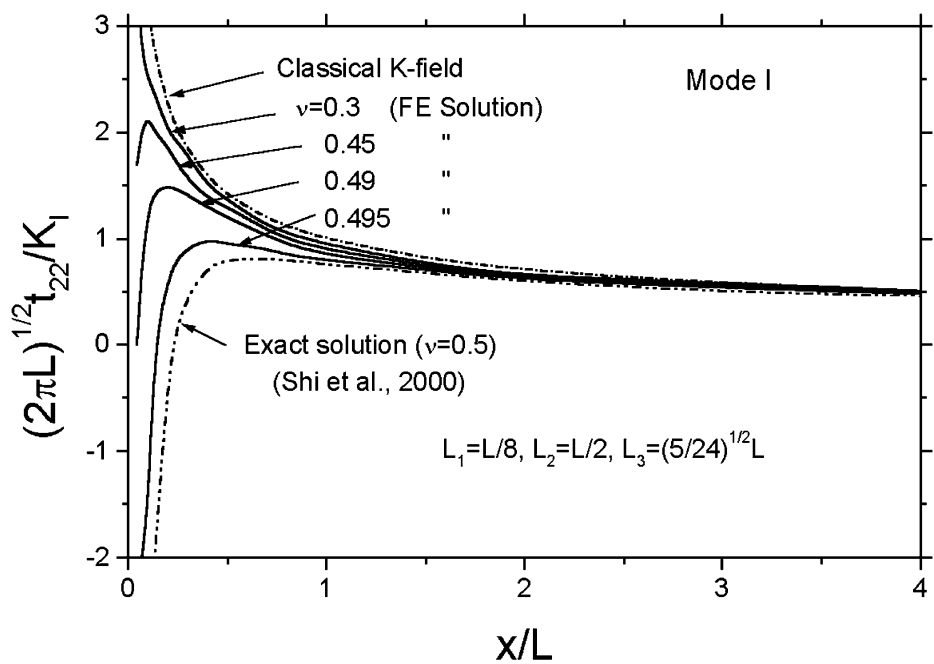

Fig. 4. Comparison of new finite element method result with exact solution for Mode I fracture. The exact solution is for the incompressible material. 
properties gradually transfer from the general compressible case to the incompressible case, the traction feature on the surface ahead of the crack tip will change from tensile to compression within the region very close to the tip. The exact solution for the incompressible case from Shi et al. (2000) is also shown in figure. Clearly, the new finite element solution compares also well with the exact solution. Moreover, from Fig. 4, the solutions are not sensitive to material Poisson ratio value except the case when the value approaches to 0.5.

From the finite element method testing and analyses of elastic fracture problems in strain gradient elasticity, the effectiveness of the new finite element method developed in the present research is confirmed. On the other hand, one may note that the sign of the traction on the plane ahead of the crack tip changes to negative from positive with approaching crack tip, it seems to be a contradiction to a truth. Actually, the effective scope of strain gradient theory is limited to a circular region around and away from the crack tip. This will be further discussed later.

\section{Elastic-plastic fracture of solids in strain gradient plasticity}

For the elastic-plastic fracture of solids based on the strain gradient plasticity theory, we have not had the closedform solutions in hand. However, the effectiveness of the new finite element method has been tested and confirmed in last section. The new finite element method has been proved to be a powerful method in dealing with the strain gradient problems. It is important to investigate the strain gradient effect on the plastic crack tip field for understanding the metal fractures in microscale. In this section, using the new finite element method analyzes the fundamental elasticplastic fracture problems considering the strain gradient effects. Similarly, the conventional linear fracture stress field (K-field) is taken as the initial equivalent body force, and the initial surface toque is set to zero, $\boldsymbol{M}=\mathbf{0}$. Solve the

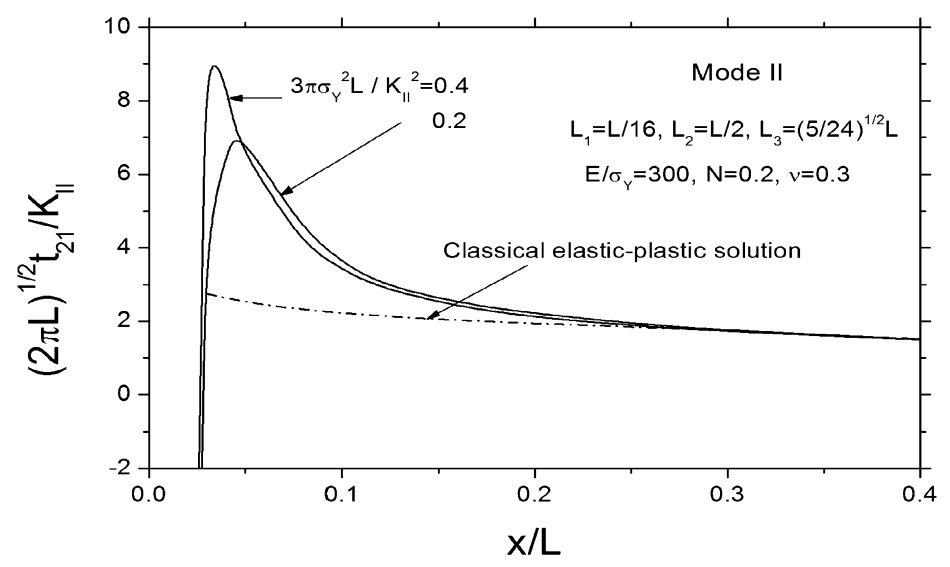

(a)

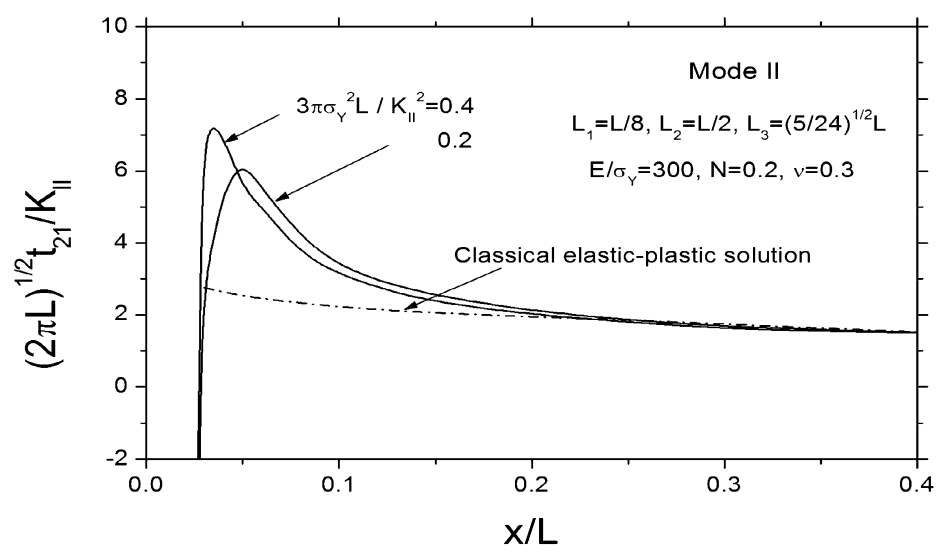

(b)

Fig. 5. Elastic-plastic fracture results for Mode II case. (a) and (b) for different length parameter compositions. 
finite element equation (30) through iteration. The elastic-plastic fracture features of the Mode I and Mode II fracture problems will be investigated, and the traction (formula (46)) distributions along the plane ahead of the crack tip will be calculated.

For easy comparison with the solutions of the strain gradient elasticity, in this section adopt the same two groups of the length parameter values as considered in last section, (1) $L_{1}=L / 16, L_{2}=L / 2, L_{3}=\sqrt{5 / 24} L$; (2) $L_{1}=L / 8$, $L_{2}=L / 2, L_{3}=\sqrt{5 / 24} L$. The first and second groups of the length parameters describe a weak and a medium stretch effects of the strain gradients, respectively.

\subsection{Mode II elastic-plastic fracture}

Fig. 5 shows the new finite element solutions for Mode II case. Comparing this results with the strain gradient elastic results shown in Fig. 3, obviously the strain gradient plasticity effect is much higher than strain gradient elasticity effect within the region $0.05 \leqslant x / L \leqslant 0.3$. Due to the strain gradient plasticity effect, the traction ahead of the tip undergoes a substantial increase. The solutions shown in Fig. 5 (a) and (b) correspond to two set selections of the length parameters, respectively. Comparing the results of Fig. 5 (a) and (b), the shear traction on the plane ahead of the crack tip decreases as parameter $L_{1}$ increases when other parameters are fixed. For comparison, the classical elastic-plastic solution is also plotted in the figures. From Fig. 5 (a) and (b), as $x$ decreases (tends to the crack tip), the shear stress $t_{21}$ increases. The solution considering the strain gradient effect is higher than the classical elastic-plastic solution. However, within a small region very near the crack tip, $x / L<0.03$, the shear traction sharply goes down

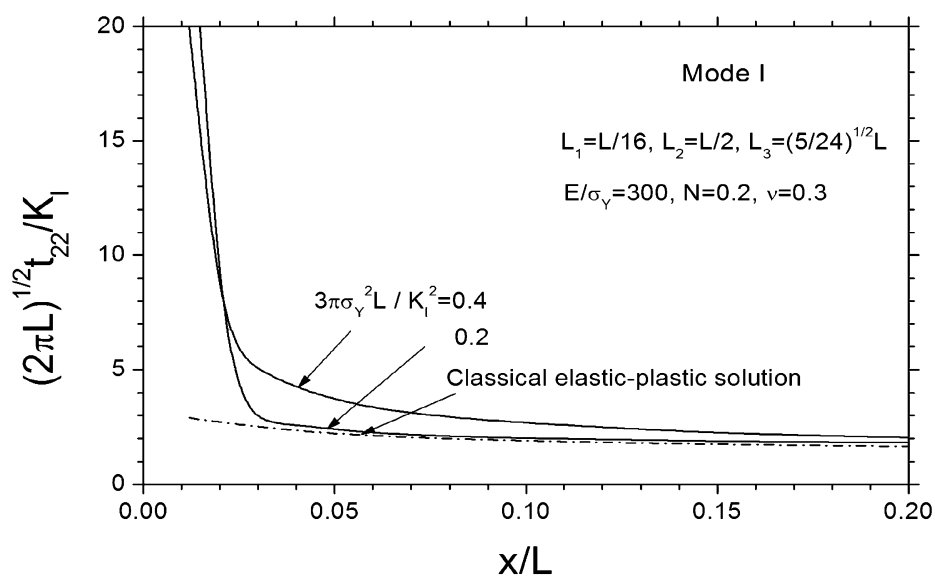

(a)

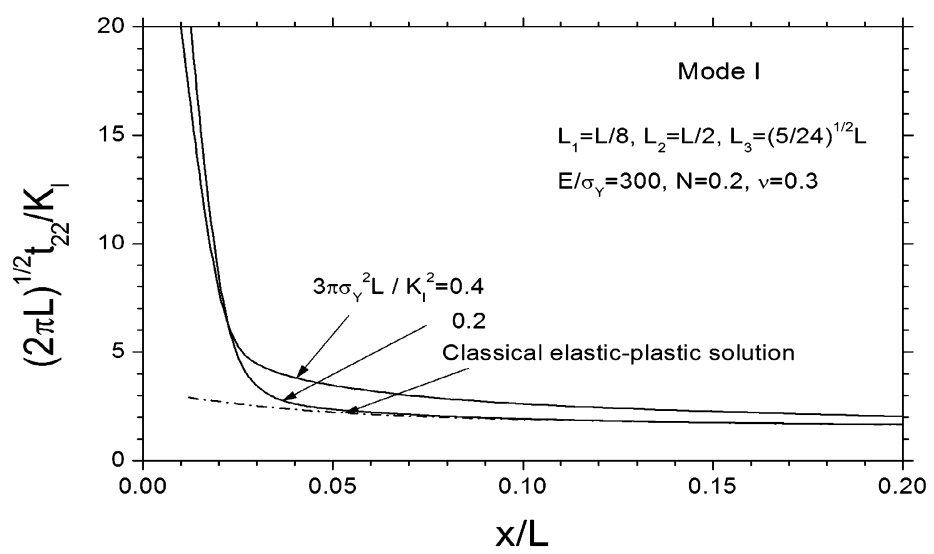

(b)

Fig. 6. Elastic-plastic fracture results for Mode I case. (a) and (b) for different length parameter selections. 
and changes sign to negative value. It seems to be a contradiction to the truth, however, the scale of this region is much smaller beyond the continuum theory attention.

\subsection{Mode I elastic-plastic fracture}

Fig. 6 shows the new finite element solutions, traction distributions along the surface ahead of the crack tip for Mode I case. The results shown in Fig. 6 (a) and (b) correspond to two group selections of the length parameters. In the figures, the variations of the normalized traction with the normalized coordinate are plotted. As the crack tip approaches, the traction increases sharply and its value is very high near the crack tip. The solution considering the strain gradient effect is much higher than the classical elastic-plastic solution.

In order to display the strain gradient effect clearly, new normalizing quantities, $\sigma_{Y}$ and $R_{P}=\left(K_{I} / \sigma_{Y}\right)^{2} / 3 \pi$, are adopted for traction and coordinates, respectively. The significance of $R_{P}$ is about the plastic zone size under the small scale yielding. The result shown in Fig. 7 is from the results in Fig. 6 (b) in the new normalizing quantities. For comparison, in Fig. 7, conventional plastic theory result is also shown in dashed line. From Fig. 7, the strain gradient plastic effect is evident when $x<0.04 R_{P}$. Using the strain gradient plasticity theory, the predicted traction near crack tip has a very high value, while using the conventional elastic-plastic theory the traction value is quite low. With increasing the stretch effect, i.e., with increasing the parameter $L_{1}$, the predicted traction along the plane ahead of the crack tip increases very much (see Fig. 8 through comparing Figs. 7 and 8). However, within a very small region

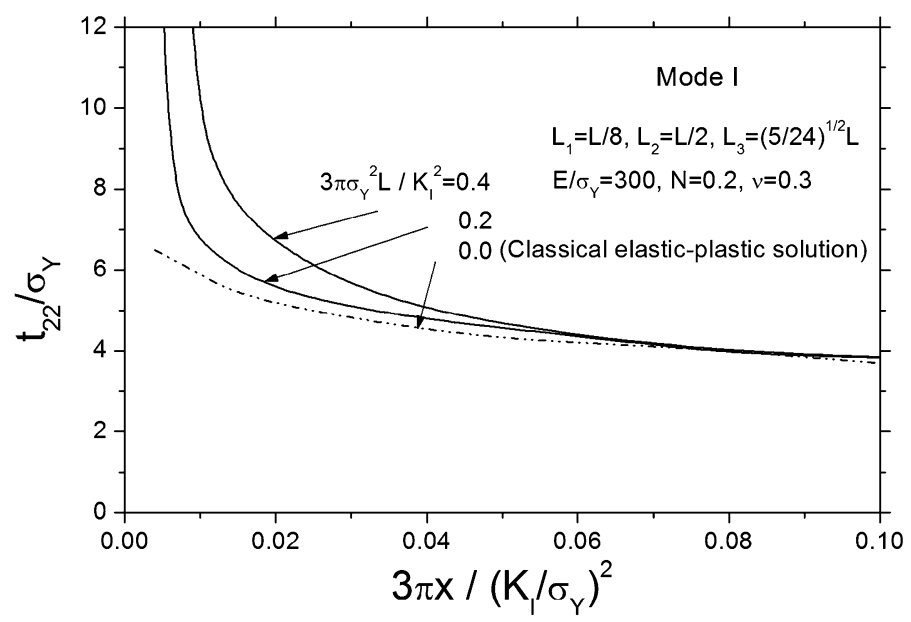

Fig. 7. Traction distributions along the plane ahead of the crack tip for several length parameter values in Mode I case.

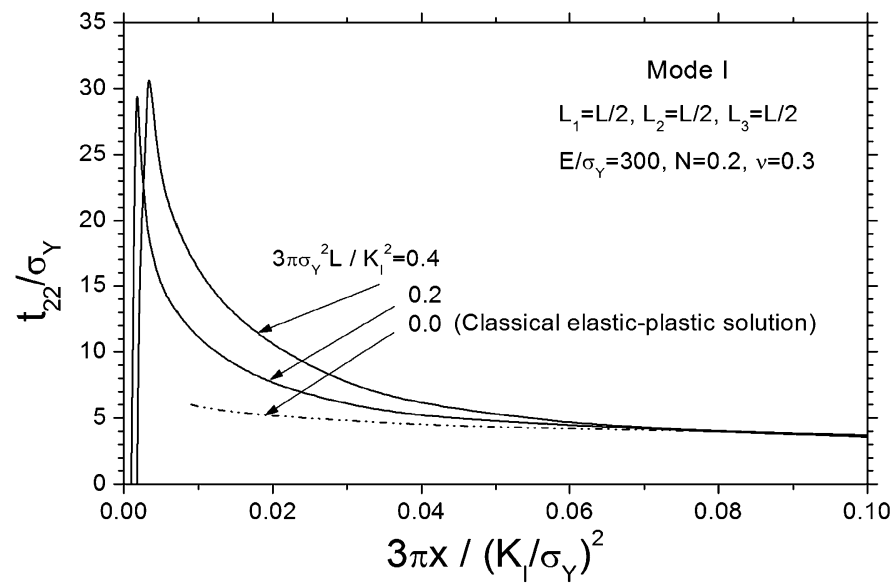

Fig. 8. Traction distributions along the plane ahead of the crack tip for several length parameter values in Mode I case. 
much close to the crack tip, the traction sign changes to negative. As stated above, the region scale of the negative traction occupation is much smaller beyond the concern of the continuum theory.

For the elastic-plastic fracture problems considering strain gradient effects, we have ever adopted the isoparametrical displacement element with nine nodes to calculate them, and have compared the results with the new finite element method results. We have found that for both Mode II and Mode III cases (shear-dominated strain gradient effects), both the iso-parametrical displacement element results and the new finite element results for each case have a big difference, however, for Mode I case (both stretch and rotation-dominated problems), both finite element results are considerably consistent with each other.

\section{Concluding remarks}

The new finite element method has been presented for strain gradient theories and has been tested to be a powerful method. In the new finite element method, the pure displacement derivatives are taken as the fundamental variables. During solving a strain gradient problem, the corresponding conventional theory stress solution is taken as the initial body force. The boundary conditions are satisfied through iteration. These make the analyses of the strain gradient problem be considerably simplified. The new finite element method concerns only on the displacement gradient terms, and is not related directly to the displacements, so that the new finite element method solutions directly supply the strains, stresses, higher-order strains and stresses. Certainly, one can obtain the displacement field through integration after the new finite element method solutions are attained. On the other hand, the new finite element method can also be applied to the analyses for the problems of the displacement boundary conditions. In this case, two calculation steps are needed: The first step is to perform a conventional elastic-plastic finite element calculation. The second step is to carry out the new finite element analysis within a zone around the crack tip, or the interface, etc. when the conventional finite element solution obtained from the first step is exerted on the boundary of the zone, in which the strain gradient effects occur within the zone.

From the analyses for mode I and mode II crack problems by using the new finite element method when strain gradient plasticity effects are considered, the traction on the plane ahead of the crack tip obtains a very high value within a region near the crack tip. This trends appear to go a long way towards overcoming the limitations of the conventional elastic-plastic theory and linking macro- and microscopic fracture behavior to atomistic fracture processes in ductile metals. However, further efforts are still required to understand the relationship between crack tip stresses and constitutive behavior in the regime near the tip where strain gradients become important, and further to understand the stress wave phenomenon (changing sign from positive to negative with approaching the crack tip).

\section{Acknowledgements}

The work is supported by National Science Foundations of China through Grants Nos. 19925211, 10432050 and 10428207; and jointly supported by Chinese Academy of Sciences through "Bai Ren” Project.

\section{References}

Aifantis, E.C., 1984. On the microstructural origin of certain inelastic models. Trans. ASME J. Engrg. Mater. Tech. 106, 326-330.

Bagchi, A., Evans, A.G., 1996. The mechanics and physics of thin film decohesion and its measurement. Interface Sci. 3, $169-193$.

Begley, M.R., Hutchinson, J.W., 1998. The mechanics of size-dependent indentation. J. Mech. Phys. Solids 46, $2049-2068$.

Chen, S.H., Wang, T.C., 2001. Strain gradient theory with couple stress for crystalline solids. Eur. J. Mech. A Solids 20, 739-756.

Engel, G., Garikipati, K., Hughes, T.J.R., Larson, M.G., Mazzei, L., Taylor, R.L., 2002. Continuous/discontinuous finite element approximations of fourth-order elliptic problems in structural and continuum mechanics with applications to thin beams and plates, and strain gradient elasticity. Comput. Methods Appl. Mech. Engrg. 191, 3669-3750.

Fleck, N.A., Muller, G.M., Ashby, M.F., Hutchinson, J.W., 1994. Strain gradient plasticity: theory and experiments. Acta Metall. Mater. 42, 475487.

Fleck, N.A., Hutchinson, J.W., 1993. A phenomenological theory for strain gradient effects in plasticity. J. Mech. Phys. Solids 41, $1825-1857$.

Fleck, N.A., Hutchinson, J.W., 1997. Strain gradient plasticity. Adv. Appl. Mech. 33, 295-361.

Gao, H., Huang, Y., Nix, W.D., Hutchinson, J.W., 1999. Mechanism-based strain gradient plasticity — I. Theory. J. Mech. Phys. Solids 47, 12391263.

Huang, Y., Chen, J.Y., Guo, T.F., Zhang, L., Hwang, K.C., 1999. Analytic and numerical studies on mode I and mode II fracture in elastic-plastic materials with strain gradient effects. Int. J. Fracture 100, 1-27. 
Lipkin, D.M., Clarke, D.R., Evans, A.G., 1998. Effect of interfacial carbon on adhesion and toughness of gold- sapphire interface. Acta Mater. 46, 4835-4850.

Ma, Q., Clarke, D.R., 1995. Size dependent hardness of solver single crystals. J. Mater. Res. 10, 853-863.

Matsushima, T., Chambon, R., Caillerie, D., 2002. Large strain finite element analysis of a local second gradient model: Application to localization. Inter. J. Numer. Methods Engrg. 54, 499-521.

McElhaney, K.W., Vlassak, J.J., Nix, W.D., 1998. Determination of indenter tip geometry and indentation contact area for depth-sensing indentation experiments. J. Mater. Res. 13, 1300-1306.

Shi, M.X., Huang, Y., Hwang, K.C., 2000. Fracture in a higher-order elastic continuum. J. Mech. Phys. Solids 48, $2513-2538$.

Shu, J.Y., Fleck, N.A., 1998. The prediction of a size effect in micro indentation. Int. J. Solids Struct. 35, 1363-1383.

Shu, J.Y., King, W.E., Fleck, N.A., 1999. Finite elements for materials with strain gradient effects. Inter. J. Numer. Methods Engrg. 44, $373-391$.

Specht, B., 1988. Modified shape functions for the three-node plate bending element passing the patch test. Inter. J. Numer. Methods Engrg. 26, $705-715$.

Stelmashenko, N.A., Walls, M.G., Brown, L.M., Milman, Y.V., 1993. Microindentations on W and Mo oriented single crystals: An STM study. Acta Metall. Mater. 41, 2855-2865.

Stolken, J.S., Evans, A.G., 1998. A microbend test method for measuring the plasticity length scale. Acta Mater. 46, 5109-5115.

Wei, Y., 2001. Particulate size effects in the particle-reinforced metal matrix composites. Acta Mech. Sinica 17, 45-58.

Wei, Y., Hutchinson, J.W., 1997. Steady-state crack growth and work of fracture for solids characterized by strain gradient plasticity. J. Mech. Phys. Solids 45, 1253-1273.

Wei, Y., Wang, X., Wu, X., Bai, Y., 2001. Theoretical and experimental researches of size effect in micro- indentation test. Sci. China Ser. A 44, $74-82$.

Xia, Z.C., Hutchinson, J.W., 1996. Crack tip fields in strain gradient plasticity. J. Mech. Phys. Solids 44, 1621-1648.

Zhang, L., Huang, Y., Chen, J.Y., Hwang, K.C., 1998. The mode III full-field solution in elastic materials with strain gradient effects. Int. J. Fracture, $325-348$.

Zienkiewicz, O.C., Taylor, R.L., 1989a. The Finite Element Method, vol. 1. Basic Formulation and Linear Problems, fourth ed. McGraw-Hill, London.

Zienkiewicz, O.C., Taylor, R.L., 1989b. The Finite Element Method, vol. 2: Solid and Fluid Mechanics, Dynamics and Non-Linearity, fourth ed. McGraw-Hill, London. 